\title{
Synchronous and bilateral oncocytic carcinoma of the breast: A case report and review of the literature
}

\author{
HIROKO ITAGAKI ${ }^{1,2}$, TOMOKO YAMAMOTO ${ }^{1}$, ATSUKO HIROI $^{1}$, KUNIO KAWANISHI $^{1}$, EIICHIRO NOGUCHI ${ }^{3}$, \\ TETSUYA OHCHI $^{3}$, TAKAKO KAMIO ${ }^{3}$, SHINGO KAMEOKA $^{3}$, HIDEAKI ODA $^{2}$ and YOJI NAGASHIMA ${ }^{1}$
}

Departments of ${ }^{1}$ Surgical Pathology, ${ }^{2}$ Pathology and ${ }^{3}$ Surgery II, Tokyo Women's Medical University, Tokyo 162-8666, Japan

Received April 24, 2015; Accepted August 10, 2016

DOI: $10.3892 / \mathrm{ol} .2017 .5610$

\begin{abstract}
Synchronous bilateral breast cancer is rare, and oncocytic carcinoma is an even rarer breast cancer histological subtype. In general, oncocytic tumors are defined as neoplasms with eosinophilic granular cytoplasm and have been reported in various organs. Oncocytic carcinoma of the breast was first documented by Gădăleanu and Craciun in 1987, and 48 cases have since been reported. The present study reports a case of synchronous bilateral breast oncocytic carcinoma. The patient was a 78-year-old woman. Although she exhibited no symptoms, chest computed tomography revealed three multinodular breast tumors: Two in the right breast and one in the left. Core needle biopsy was performed on the three tumors, and the patient was diagnosed with invasive ductal carcinoma with potential apocrine carcinoma. A bilateral modified radical mastectomy was performed. Surgical specimens of the three tumors revealed cord- or nest-forming tumor cells with eosinophilic granular cytoplasm. Immunohistochemically, the tumor cells were markedly positive for mitochondria. Electron microscopy of the tumor samples additionally revealed numerous mitochondria filling the cytoplasm. Based on these findings, the tumors were diagnosed as oncocytic carcinoma. The pathogenesis of oncocytic carcinoma remains to be fully elucidated; thus, additional clinicopathological studies are required.
\end{abstract}

\section{Introduction}

Oncocytic tumors, including oncocytomas and oncocytic carcinomas, are comprised of oncocytic cells. The term 'oncocyte' was initially used to refer to a cellular change in the salivary glands by Hamperl in 1931 (1). Oncocytes are cells with an eosinophilic granular and reticular cytoplasm and, in the majority of cases, immunohistological analysis by light

Correspondence to: Dr Hiroko Itagaki, Department of Surgical Pathology, Tokyo Women's Medical University, 8-1 Kawada-Cho, Shinjuku-Ku, Tokyo 162-8666, Japan

E-mail: hitagaki@research.twmu.ac.jp

Key words: oncocytic carcinoma, breast, mitochondria, bilateral, synchronous microscopy reveals that $>60 \%$ of the cytoplasm is occupied by mitochondria (2). Oncocytic tumors are occasionally observed in the salivary gland, thyroid gland, kidney, parathyroid gland and pituitary gland (3-8).

Oncocytomas and oncocytic carcinomas, which are commonly used terms for benign and malignant tumors, respectively (3), are comprised of oncocytic cells. Oncocytic carcinoma of the breasts is extremely rare (9). It was first reported by Gădăleanu and Craciun in 1987 (10), and 48 cases have since been reported, 15 of which were diagnosed retrospectively (9).

Oncocytes and apocrine cells exhibit similar appearances following hematoxylin and eosin staining. Apocrine metaplasia is frequently observed in breast lesions. When eosinophilic tumor cells are observed, a diagnosis of oncocytic carcinoma should be considered, and immunohistochemical or electron microscopic analysis is advisable $(2,11,12)$. A previous study reported that the clinical features of oncocytic carcinoma are similar to those of other types of invasive carcinomas (9).

The present study reports a case of synchronous bilateral oncocytic carcinoma of the breast, which is extremely rare but is considered important to the pathogenesis of oncocytic carcinoma.

\section{Case report}

A 78-year-old Japanese woman exhibited bilateral breast tumors on chest computed tomography (CT) during surveillance following an operation for colon cancer. The patient's medical history included non-insulin-dependent diabetes, acromegaly, hypertension and colon cancer. Six months previously, the patient had been diagnosed with colon cancer, and an ileocecal resection was performed. Adjuvant chemotherapy with $300 \mathrm{mg}$ /day oral tegafur/uracil plus $75 \mathrm{mg} /$ day calcium folinate (Taiho Pharmaceutical, Co., Ltd., Tokyo, Japan) was administered for 4 weeks (including a 1 week rest) and continued for a total of four cycles. Contrast-enhanced chest CT revealed three hyperdense masses in the breasts: Two in the right breast and one in the left (Fig. 1). Core needle biopsies were performed on the three tumors. Tumor cells with eosinophilic cytoplasm were identified in all three lesions (Fig. 2). The tumors were diagnosed as invasive ductal carcinoma, with potential apocrine carcinoma. As 

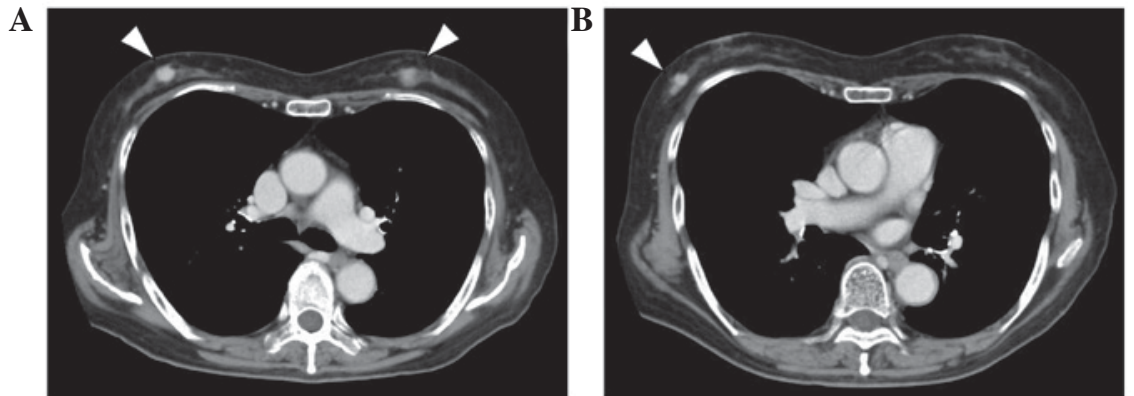

Figure 1. Contrast-enhanced computed tomography (CT). (A) CT scans revealed bilateral enhanced solid tumors (white arrowheads). (B) An additional tumor was revealed in the right breast (arrowhead).
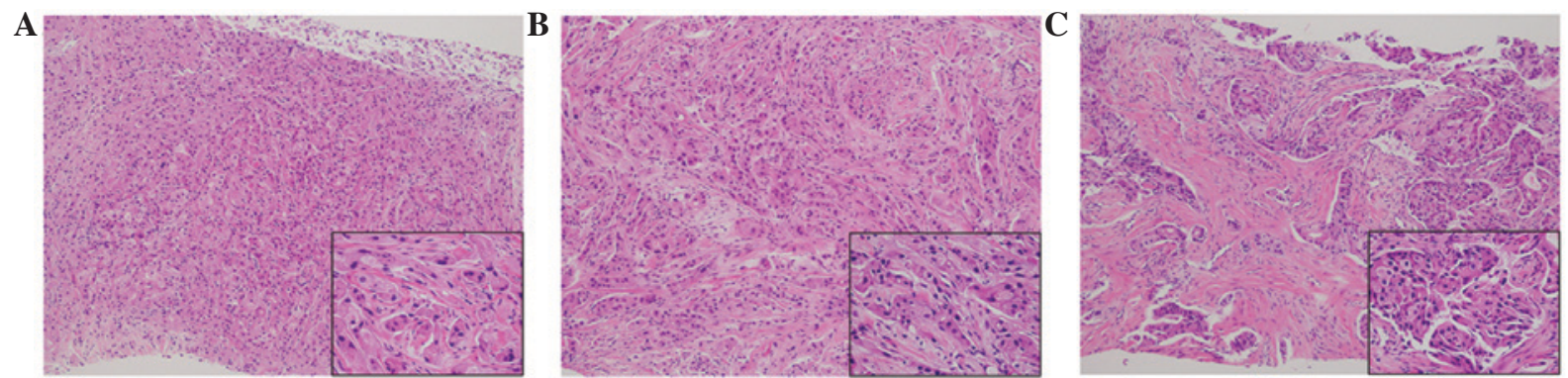

Figure 2. Histopathological findings of the core needle biopsy. Hematoxylin and eosin staining of the (A) right upper outer quadrant, (B) right outer quadrant and (C) left upper inner quadrant identified tumor cells with eosinophilic cytoplasms in all three lesions. Main image magnification, x100; inset panel magnification, $\mathrm{x} 400$.

no metastasis was detected radiologically, the patient was treated with a bilateral modified radical mastectomy and sentinel lymph node biopsy in a single session. Intraoperative pathological diagnosis showed no metastasis in the sentinel nodes. The patient did not undergo further treatment and was followed-up every 3 months by blood and imaging examinations. She remained free of relapse and metastasis for 7 months after the surgery.

Macroscopic examination of the resected breasts revealed three white solid masses: One in the right upper outer quadrant $(13 \times 12 \times 10 \mathrm{~mm})$, one in the right outer quadrant $(10 \times 7 \times 10 \mathrm{~mm})$ and one in the left upper inner quadrant (17x15x12 mm) (Fig. 3). Histologically, all of the tumors were comprised of solid cell sheets and nests of tumor cells. The tumor cells possessed abundant eosinophilic granular cytoplasm (Fig. 4). Invasion into the fat was observed in the right breast.

Immunohistochemical analysis was performed using an autostainer (Ventana Medical Systems, Inc. Tucson, AZ, USA). Breast tissue sections $(4-\mu \mathrm{m})$ were incubated with mouse anti-cytokeratin-7 (1:200; cat. no. M7018; Dako, Glostrup, Denmark), mouse anti-epithelial membrane antigen (1:100; cat. no. 247M-96; Cell Marque Corporation, Rocklin, CA, USA), mouse anti-E-cadherin (1:100; cat. no. M3612; Dako), mouse anti-gross cystic disease fluid protein-15 (1:50; cat. no. SIG-3611-1000; Covance, Inc., Princeton, NJ, USA), mouse anti-mitochondria (1:500; cat. no. B-MU213UC; BioGenex, San Ramon, CA, USA), rabbit anti-estrogen receptor (prediluted; cat. no. 518-107925; Roche Diagnostics, Basel, Switzerland), rabbit anti-progesterone receptor (prediluted; cat . no. 790-2223; Roche Diagnostics) and anti-human epidermal growth factor receptor 2 (prediluted; cat. no. 518107918; Roche

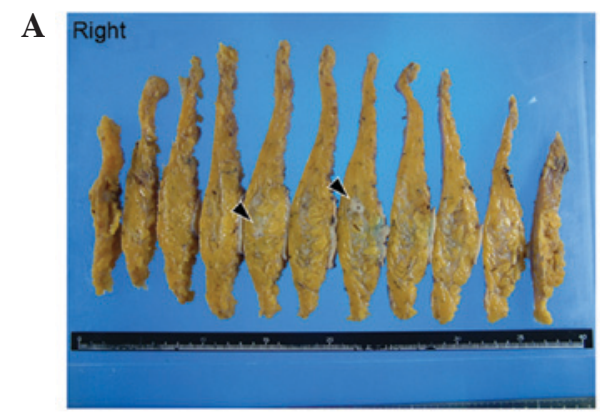

B

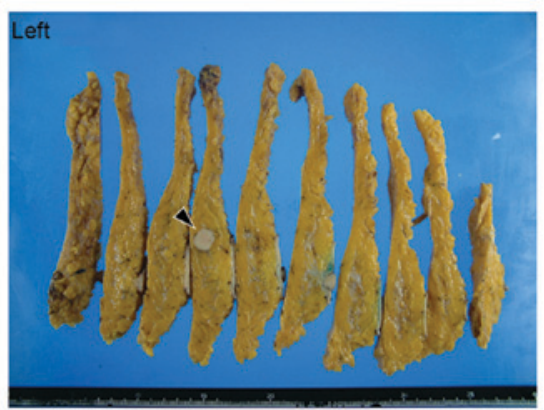

Figure 3. Macroscopic appearance of the surgical specimen. Three solid tumors were observed. (A) Two tumors were present in the right breast and (B) one tumor was present in the left breast (black arrowheads).

Diagnostics) antibodies. The primary antibodies were visualized using horseradish peroxidase-conjugated secondary antibodies and the ultraView Universal DAB Detection kit (cat. no. 760-500; Ventana Medical Systems, Inc.), after which the sections were counterstained for $1 \mathrm{~min}$ with Carrazzi's 

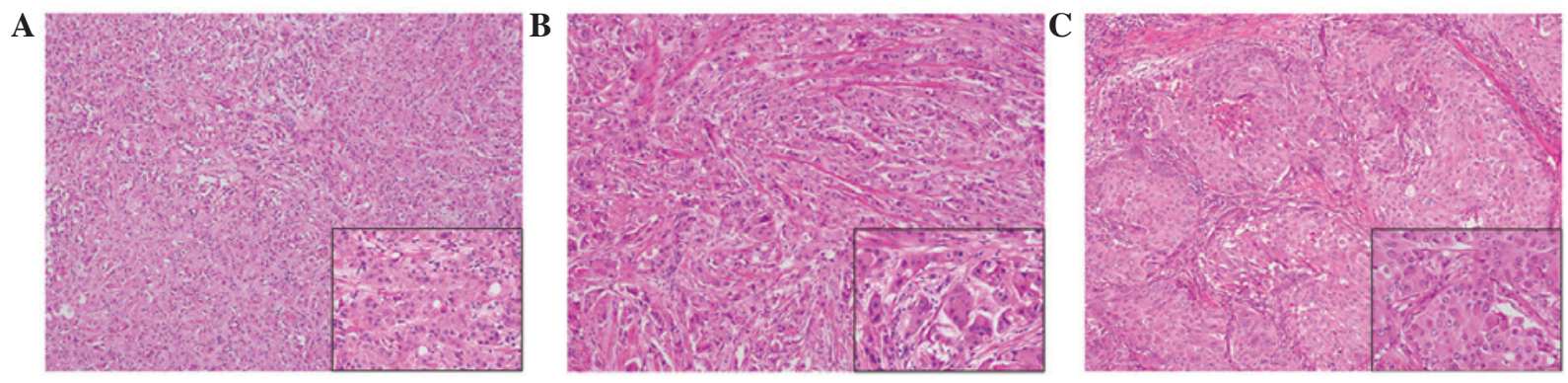

Figure 4. Histopathological findings of the surgical specimens. Hematoxylin and eosin staining of the (A) right upper outer quadrant, (B) right outer quadrant and (C) left upper inner quadrant revealed tumor cells with abundant eosinophilic granular cytoplasms in all three tumors. Main image magnification, x100; inset panel magnification, $\mathrm{x} 400$.
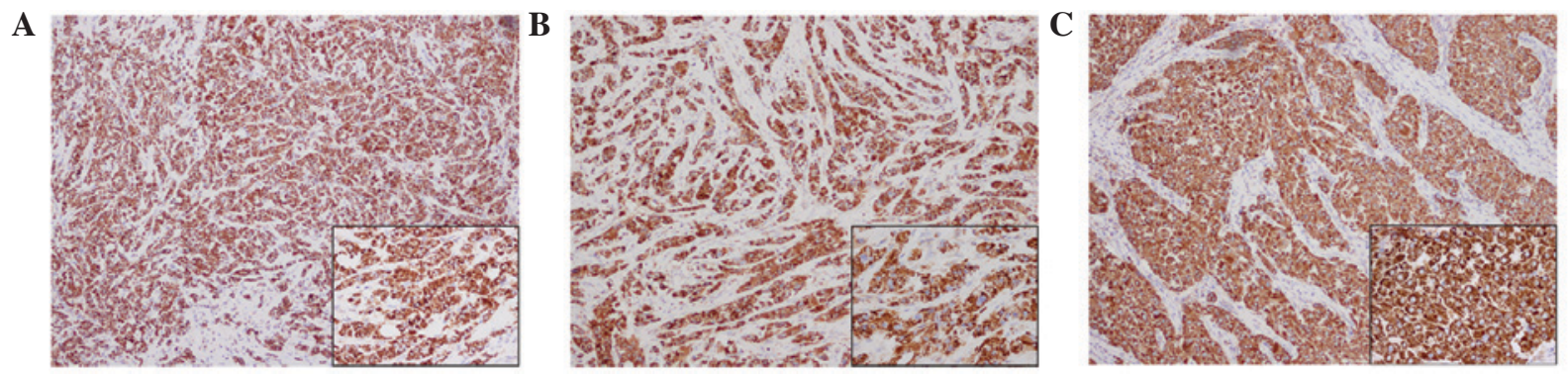

Figure 5. Immunohistochemical staining of the surgical specimens. More than $70 \%$ of the tumor cells were strongly positive for mitochondria. (A) Right upper outer quadrant. (B) Right outer quadrant. (C) Left upper inner quadrant. Mitochondrial staining with an anti-mitochondria antibody. Main image magnification, $\mathrm{x} 100$; inset panel magnification, $\mathrm{x} 400$.

hematoxylin solution. Immunohistochemically, the tumor cells were positive for cytokeratin-7, epithelial membrane antigen and E-cadherin (data not shown). Additionally, gross cystic disease fluid protein-15 showed a diffuse and weakly positive reaction (data not shown). Notably, all three tumors were strongly positive for mitochondria in the majority of the tumor cells (Fig. 5). The peritumoral lesion contained ductal epithelia with eosinophilic cytoplasm, which also demonstrated strong reactivity for anti-mitochondrial antibody (Fig. 6). The tumor cells were negative for estrogen and progesterone receptors. Human epidermal growth factor receptor 2 was weakly positive along the membrane and was scored $1+$ using the HercepTest (Dako), according to the manufacturer's protocol (data not shown) (13). Electron microscopy performed on formalin-fixed tumor tissue revealed numerous mitochondria (Fig. 7). Based on these findings, the tumors were diagnosed as synchronous bilateral oncocytic carcinoma of the breast.

\section{Discussion}

Oncocytic carcinoma of the breast is defined as a tumor in which $>70 \%$ of tumor cells demonstrate oncocytic characteristics, and is classified as 'uncommon' according to the World Health Organization classification (14). To the best of our knowledge, only six cases of oncocytic carcinoma of the breast had been previously reported before Ragazzi et al reviewed 32 cases in $2011(9-11,15,16)$. The tumor is characterized by oncocytic tumor cells containing numerous mitochondria (14).

Candidates for the differential diagnosis of oncocytic carcinoma include ductal carcinoma with partial apocrine differentiation, acinic cell carcinoma, apocrine carcinoma, granular cell tumors and metastatic carcinoma arising from eosinophilic granular cytoplasm $(17,18)$. On routine hematoxylin and eosin staining of specimens, it is difficult to recognize eosinophilic cells as oncocytes (19). Immunohistochemical or electron microscopic analyses are required to distinguish these neoplastic cells $(3,9)$. Apocrine metaplasia is frequently observed in breast lesions (20). The cells of apocrine metaplasia typically exhibit abundant eosinophilic cytoplasm containing brightly eosinophilic granules, and secretory 'snouts' are typically observed by light microscopy (20). Ultrastructurally, the cytoplasm of granular cell tumors is packed with numerous lysosomes, and apocrine cells contain abundant granules surrounding the nuclei $(12,19)$. Oncocytic carcinoma is characterized by strong immunopositivity for mitochondria, and numerous mitochondria fill the cytoplasm when examined by electron microscopy (2). The present study reported a case involving a patient with synchronous and bilateral oncocytic carcinoma. In the present case, immunohistochemistry for mitochondria revealed markedly positive signals in the cytoplasm, and electron microscopy demonstrated large numbers of mitochondria. These findings supported the diagnosis of oncocytic carcinoma of the breast.

Oncocytic cells are occasionally observed in glandular epithelia that have high metabolic activity, including that in the salivary glands, thyroid gland, kidney, parathyroid gland and pituitary gland (3-8). However, the mechanism of oncocytic change remains to be fully elucidated; it has been reported that oncocytic change may be a type of senescent change. Previous studies have noted that increased oncocytic changes in the salivary glands and liver are associated with senescence and the administration of certain drugs (21-23). Frequent 

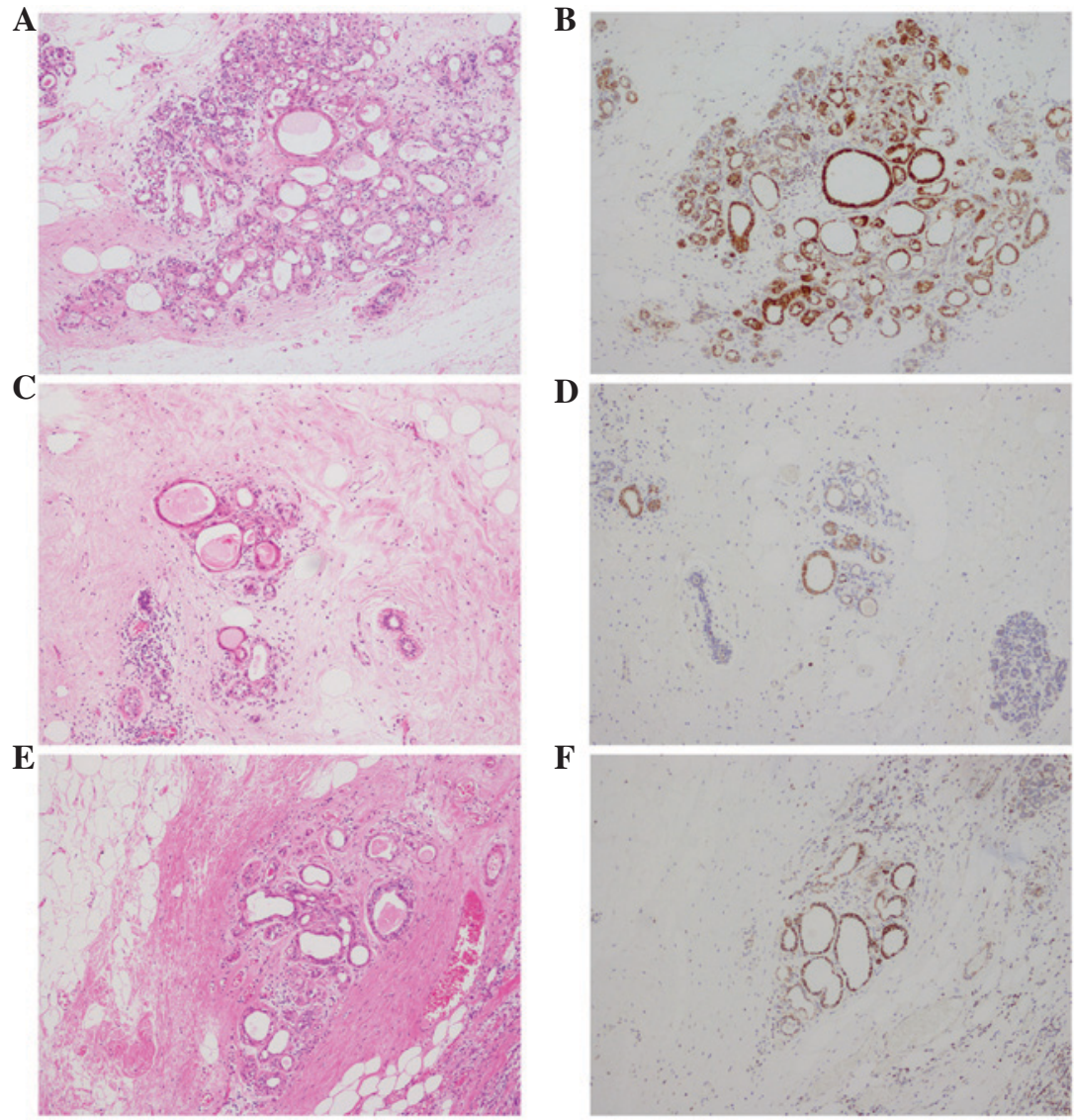

Figure 6. Immunohistochemical staining of the surgical specimens counterstained with hematoxylin and eosin (HE). Oncocytic cells were observed in the peritumoral lesion, which also showed reactivity for anti-mitochondrial antibody. (A) Right upper outer quadrant. HE staining. (B) Right upper outer quadrant. Mitochondrial staining. (C) Right outer quadrant. HE staining. (D) Right outer quadrant. Mitochondrial staining. (E) Left upper inner quadrant. HE staining. (F) Left upper inner quadrant. Mitochondrial staining. Magnification, x100.

mitochondrial DNA abnormalities have been observed in oncocytic lesions in the thyroid gland, kidney, salivary glands, adrenal cortex and parathyroid gland $(6,24)$. A small number of cases in the thyroid have exhibited mutations in nuclear DNA genes encoding oxidative phosphorylation proteins (24). Geyer et al (25) reported chromosomal changes in oncocytic carcinoma of the breast. Oncocytic carcinoma of the breast frequently exhibits gains of 11q13.1-13.2 and 19p13, similar to oncocytic tumors of the kidney and thyroid. In the present case, non-neoplastic duct epithelia adjacent to the tumor demonstrated oncocytic changes. Although gene mutations were not analyzed in the present case, the oncocytic carcinoma of the breast may have been derived from ducts with oncocytic changes.

A review of the literature reveals that the clinical features of oncocytic carcinomas are similar to those of invasive ductal carcinoma, not otherwise specified (9). Thus, the therapeutic strategies are identical. However, to the best of our knowledge, no previous reports have described the use and effects of radiation therapy for the treatment of oncocytic carcinoma of the breast, due to the rarity of this tumor. Resistance to radiotherapy has been reported in oncocytic tumors at other sites, including the rectum and meninges $(26,27)$. Thus, certain modifications may be required to utilize radiotherapy in the treatment of oncocytic carcinoma of the breast.

Bilateral breast cancer is uncommon and represents 2-6\% of all breast carcinomas (28). Synchronous carcinoma is defined

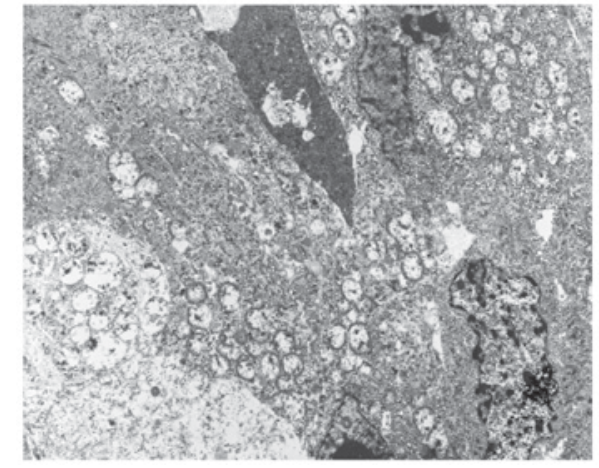

Figure 7. Ultrastructural features of the oncocytic carcinoma, as determined by electron microscopy. Mitochondria were scattered throughout the cytoplasm. Magnification, x3,000.

as a second cancer diagnosed within 3 months of the diagnosis of a first cancer (28). Although both synchronous breast cancer and oncocytic carcinoma of the breast are uncommon, synchronous bilateral oncocytic carcinoma of the breast is extremely rare $(9,29,30)$. Thorough follow-up is important, and additional clinicopathological studies are required to analyze effective treatments for oncocytic carcinoma.

In conclusion, the present study demonstrated that it is difficult to diagnose oncocytic carcinoma of the breast merely by light microscopy. To distinguish breast neoplasms 
composed of tumor cells with abundant eosinophilic cytoplasms from oncocytic carcinoma, immunohistochemical or electron microscopy analyses should be performed.

\section{References}

1. Hamperl $\mathrm{H}$ : Beiträge zur normalen und pathologischen Histologie menschlieher Speicheldriisen. Z Mikrosk Anat Forsch 27: 1-55, 1931 (In German).

2. Ghadially FN (ed): Diagnostic electron microscopy of tumours. 2nd edition. Butterworth \& Company, London, 1985.

3. Tallini G: Oncocytic tumours. Virchows Arch 433: 5-12, 1998

4. Kim YL, Jang YW, Kim JT, Sung SA, Lee TS, Lee WM and Kim HJ: A rare case of primary hyperparathyroidism associated with primary aldosteronism, Hürthle cell thyroid cancer and meningioma. J Korean Med Sci 27: 560-564, 2012.

5. Hoang MP, Ayala AG and Albores-Saavedra J: Oncocytic adrenocortical carcinoma: A morphologic, immunohistochemical and ultrastructural study of four cases. Mod Pathol 15: 973-978, 2002

6. Máximo V, Rios E and Sobrinho-Simões M: Oncocytic lesions of the thyroid, kidney, salivary glands, adrenal cortex, and parathyroid glands. Int J Surg Pathol 22: 33-36, 2014.

7. Yamakita N, Ikeda T, Murai T, Kimura M, Komaki T, Miura K, Iwamura M, Hirata T and Umezaki: Panhypopituitarism due to Rathke's cleft cyst associated with pituitary oncocytoma. Intern Med 36: 107-112, 1997.

8. Chakrabarti I, Basu A and Ghosh N: Oncocytic lesion of parotid gland: A dilemma for cytopathologists. J Cytol 29: 80-82, 2012

9. Ragazzi M, de Biase D, Betts CM, Farnedi A, Ramadan SS, Tallini G, Reis-Filho JS and Eusebi V: Oncocytic carcinoma of the breast: Frequency, morphology and follow-up. Hum Pathol 42: 166-175, 2011.

10. Gădăleanu V and Craciun C: Malignant oncocytoma of the breast. Zentralbl Allg Pathol 133: 279-283, 1987.

11. Damiani S, Eusebi V, Losi L, D’Adda T and Rosai J: Oncocytic carcinoma (malignant oncocytoma) of the breast. Am J Surg Pathol 22: 221-230, 1998.

12. Hashimoto K, Gross BG and Lever WF: Electron microscopic study of apocrine secretion. J Invest Dermatol 46: 378-390, 1966.

13. Wolff AC, Hammond ME, Schwartz JN, Hagerty KL, Allred DC, Cote RJ, Dowsett M, Fitzgibbons PL, Hanna WM, Langer A, et al; American Society of Clinical Oncology; College of American Pathologists: American Society of Clinical Oncology/College of American Pathologists guideline recommendations for human epidermal growth factor receptor 2 testing in breast cancer. $\mathrm{J}$ Clin Oncol 25: 118-145, 2006.

14. Lakhani SR, Ellis IO, Schnitt SJ, Tan PH and van de Vijver MJ (eds): WHO Classification of Tumours of the Breast. 4th edition. IARC Press, Lyon, 2012.

15. Hamperl H: Oncocytes and hyaline inclusions in the human breast. Virchows Arch B Cell Pathol 10: 88-92, 1972 (In German).
16. Costa MJ and Silverberg SG: Oncocytic carcinoma of the male breast. Arch Pathol Lab Med 113: 1396-1399, 1989.

17. Yamazaki M, Nagata Y, Monji S, Shigematsu Y, Baba T, Shimokawa $\mathrm{H}$, Uramoto $\mathrm{H}$, Yamada $\mathrm{S}$, Hanagiri $\mathrm{T}$ and Tanaka F: Apocrine carcinoma of the breast. J UOEH 33: 293-301, 2011.

18. Coyne JD and Dervan PA: Primary acinic cell carcinoma of the breast. J Clin Pathol 55: 545-547, 2002.

19. Chang A and Harawi SJ: Oncocytes, oncocytosis, and oncocytic tumors. Pathol Annu 27: 263-304, 1992.

20. Durham JR and Fechner RE: The histologic spectrum of apocrine lesions of the breast. Am J Clin Pathol 113 (5 Suppl 1): S3-S18, 2000.

21. Mete O and Asa SL: Oncocytes, oxyphils, Hürthle, and Askanazy cells: Morphological and molecular features of oncocytic thyroid nodules. Endocr Pathol 21: 16-24, 2010.

22. Baris O, Savagner F, Nasser V, Loriod B, Granjeaud S, Guyetant S, Franc B, Rodien P, Rohmer V, Bertucci F, et al: Transcriptional profiling reveals coordinated up-regulation of oxidative metabolism genes in thyroid oncocytic tumors. J Clin Endocrinol Metab 89: 994-1005, 2004.

23. Martinez-Madrigal F and Micheau C: Histology of the major salivary glands. Am J Surg Pathol 13: 879-899, 1989.

24. Máximo V, Botelho T, Capela J, Soares P, Lima J, Taveira A, Amaro T, Barbosa AP, Preto A, Harach HR, et al: Somatic and germline mutation in GRIM-19, a dual function gene involved in mitochondrial metabolism and cell death, is linked to mitochondrion-rich (Hurthle cell) tumours of the thyroid. Br J Cancer 92: 1892-1898, 2005.

25. Geyer FC, de Biase D, Lambros MBK, Ragazzi M, Lopez-Garcia MA, Natrajan R, Mackay A, Kurelac I, Gasparre G, Ashworth A, et al: Genomic profiling of mitochondrion-rich breast carcinoma: Chromosomal changes may be relevant for mitochondria accumulation and tumour biology. Breast Cancer Res Treat 132: 15-28, 2012.

26. Marucci G, Betts CM, Frank G and Foschini MP: Oncocytic meningioma: Report of a case with progression after radiosurgery. Int J Surg Pathol 15: 77-81, 2007.

27. Ambrosini-Spaltro A, Salvi F, Betts CM, Frezza GP, Piemontese A, Del Prete P, Baldoni C, Foschini MP and Viale G: Oncocytic modifications in rectal adenocarcinomas after radio and chemotherapy. Virchows Arch 448: 442-448, 2006.

28. Hartman M, Czene K, Reilly M, Adolfsson J, Bergh J, Adami HO, Dickman PW and Hall P: Incidence and prognosis of synchronous and metachronous bilateral breast cancer. J Clin Oncol 25: 4210-4216, 2007.

29. Chandrika, Permi HS, Kishan Prasad HL, Mohan R, Shetty KJ and Patil C: Synchronous bilateral medullary carcinoma of breast: Is it metastasis or second primary? J Cancer Res Ther 8: 129-131, 2012.

30. Adami HO, Hansen J, Jung B, Lindgren A and Rimsten A: Bilateral carcinoma of the breast. Epidemiology and histopathology. Acta Radiol Oncol 20: 305-309, 1981. 